\title{
Optimal Design and Dimensionless Correlation Development for Natural Convection Cooling Board
}

\author{
Tohru Nakanishi ${ }^{*}$ Non-member Koki Shimohashi* ${ }^{*}$ Non-member \\ Tomohiro Hase ${ }^{* *} \quad$ Member
}

This paper focuses on the many parameters of PCB (Printed Circuit Board) that might affect on heat flow in the PCB. The parameter study found some guidelines that contribute to provide effective cooling PCB structure, and the study also found that the PCB structure affects temperature distribution of the PCB. Such heat transfer brings up many factors, however dimensionless analysis is considered appropriate way to study this complex heat transfer. Non-dimensional correlation between Nusselt number $\mathrm{Nu}$ ) and Grashoff number (Gr), dedicated to high-density PCB, was derived in natural convection cooling system. For the verification of the correlation, experimental simulation was performed in basis of several PCB properties. And unique definitions were given to dimensionless numbers.

Keywords : Cooling, Convection, Board, Dimensionless number, Nusselt number, Grashoff number

\section{Introduction}

Electronic products such as mobile phone, DVC (Digital Video Camera), DSC (Digital Still Camera), PDA (Personal Device Assistant) and notebook computer, are getting their size compact. The complexity of many parameters depending on the quality is one of the technical hurdle for the purpose of the down-sizing. Therefore to achieve the high density packaging, some unique parameter study and the numerical simulation technique are introduced for the optimal structural and thermal design ${ }^{(1)(2)}$.

In the thermal area, it is a well-known measurement to put a heat sink or cooling fan for thermal management. However, these measurements cannot be adopted for these compact devices, because these compact devices are too small to put such cooling parts. It is one solution to select low power component, but it is hard to find substitute component in many cases. This story clarifies the needs of studying PCB structure that works under required conditions of size and temperature.

This paper focuses on the many parameters of PCB that might affect on heat flow in the PCB. A build-up 6-layer PCB is taken throughout this paper and the numerical simulation model of the PCB was established in basis of copper layer thickness and its remaining copper ratio, insulation layer thickness and PCB size. The parameter study found some guidelines that contribute to provide effective cooling PCB structure, and the study also found that the PCB structure affects temperature distribution of the PCB. The component and $\mathrm{PCB}$ are cooled with convection, and a part of the heat can be conducted to PCB. The temperature distribution is considered to be the result of the heat transfer.

It can be said that the final goal is to control the temperature of component to get it work under a certain environment. To reach the goal studying both of the thermal convection and temperature distribution of PCB is essential. Such heat transfer brings up many

* IBM Japan, Ltd. Yasu

800, Ichimiyake, Yasu-cho, Yasu-gun, Shiga-ken, 520-2392

** Ryukoku Universuty

1-5, Yokoya, Ohe-cho, Seta, Ohtsu-shi, Shiga-ken, 520-2194 factors, however dimensionless analysis is considered appropriate way to study this complex heat transfer.

In this paper, non-dimensional numbers correlation that can be applied to PCB cooling system is developed to evaluate the heat convection and temperature distribution. The unique definitions of non-dimensional numbers enable to express the heat transfer of PCB cooling.

\section{PCB Cross Section Structure}

Figure 1 shows the cross section of the 6-layer build up PCB. For the purpose of high density packaging, additional 2 copper layers are build up at both side of the conventional 4 internal copper layers. It is common that the internal copper plane is tried to be used as the signal line, and that line is put between ground layers for the protection against the external noise. And the outer copper layer has the higher contribution to the heat transfer.

Therefore the outermost, 2nd outermost copper layer and solder resist layer are evaluated in this paper. This 6-layer PCB structure is taken throughout this paper. Originally this PCB is designed for compact amusement game device.

\begin{tabular}{|c|c|}
\hline Solder resist & $40 \mu \mathrm{m}$ \\
\hline Copper & $48 \mu \mathrm{m}$ \\
\hline Insulation & $150 \mu \mathrm{m}$ \\
\hline Copper & $35 \mu \mathrm{m}$ \\
\hline Insulation & $100 \mu \mathrm{m}$ \\
\hline Copper & $35 \mu \mathrm{m}$ \\
\hline Insulation & $200 \mu \mathrm{m}$ \\
\hline Copper & $35 \mu \mathrm{m}$ \\
\hline Insulation & $100 \mu \mathrm{m}$ \\
\hline Copper & $35 \mu \mathrm{m}$ \\
\hline Insulation & $150 \mu \mathrm{m}$ \\
\hline Copper & $48 \mu \mathrm{m}$ \\
\hline Solder resist & $40 \mu \mathrm{m}$ \\
\hline
\end{tabular}

Fig. 1. PCB Cross section. 


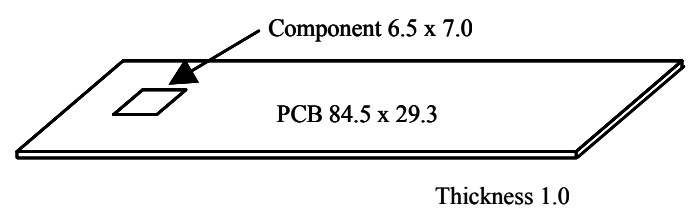

Fig. 2. Schematic diagram of computational simulation.

Table 1. Material properties.

\begin{tabular}{llll}
\hline \hline & $\begin{array}{l}\text { Thermal } \\
\text { conductivity } \\
(\mathrm{W} / \mathrm{mK})\end{array}$ & $\begin{array}{l}\text { Density } \\
\times 10^{3} \\
\left(\mathrm{~kg} / \mathrm{m}^{3}\right)\end{array}$ & $\begin{array}{l}\text { Specific } \\
\text { Heat } \times 10^{3} \\
(\mathrm{~J} / \mathrm{kgK})\end{array}$ \\
\hline $\mathrm{Cu}$ & 356 & 8.93 & 0.458 \\
Solder resist & 0.117 & 1.10 & 1.90 \\
Insulation & 0.090 & 1.20 & 2.80 \\
L1 $(30 \% \mathrm{Cu})$ & 106 & 3.45 & 1.47 \\
P1 $(80 \% \mathrm{Cu})$ & 284 & 7.39 & 0.927 \\
L3 $(9.6 \% \mathrm{Cu})$ & 34.2 & 1.95 & 2.60 \\
L4 $(7.5 \% \mathrm{Cu})$ & 26.7 & 1.78 & 2.62 \\
P2 $(75 \% \mathrm{Cu})$ & 266 & 7.00 & 1.05 \\
L6 $(15 \% \mathrm{Cu})$ & 53.4 & 2.27 & 1.69 \\
\hline
\end{tabular}

\section{Numerical Analysis}

3.1 Numerical Analysis Model A PCB that has one heat dissipate component on its top surface is placed in the air in horizontal direction shown in Figure 2. In this numerical analysis, solution domain is set around the PCB including air then analysis is conducted in steady states with natural convection.

The material properties of this model are given in Table.1. The Copper layers L1 to L6 actually consist of copper and insulation with a certain volume ratio. Each of the material properties for L1 to L6 are defined with the Copper volume ratio in each layer. The ratio is referred to as $\mathrm{Cu}$ remained ratio hereafter, which ranges from $7.5 \%$ to $80 \%$.

3.2 Verification of Numerical analysis In this section, actual hardware testing is performed in order to verify the capability of the numerical analysis model that is introduced in previous section. The numerical analysis based on the model is performed with the following boundary conditions. The temperature of the component $T_{\text {ref }}$ is 56.5 degree $\mathrm{C}$, and the ambient temperature $T_{a m b}$ is 23.0 degree $\mathrm{C}$.

As to the hardware testing, a PCB is prepared that has the same cross-section structure and board size as those of numerical analysis model. The PCB is placed in the air in the same manner as the numerical analysis. The hatching area in Figure 3 is heated with heater, where the outer most copper layer is exposed to the air. After taking the sufficient time to make the system steady states, seven surface points [a] to [g] and hatching area shown in Figure 3 are measured with thermo-couple. The all measurement points are on the PCB surface.

The calculated temperature distributions by the numerical analysis are shown in Figure 4. All the measured temperature by the hardware testing at each measurement points and calculated numerical analysis are given in Table 2. The calculated temperature distribution Figure 4 shows that the heat from the component is conducted in the PCB and cooled by convection

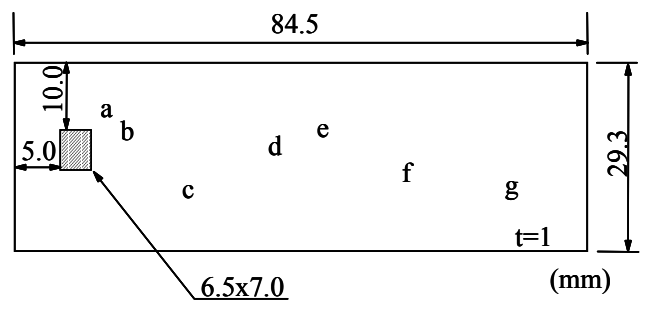

Fig. 3. Temperature measurement points.

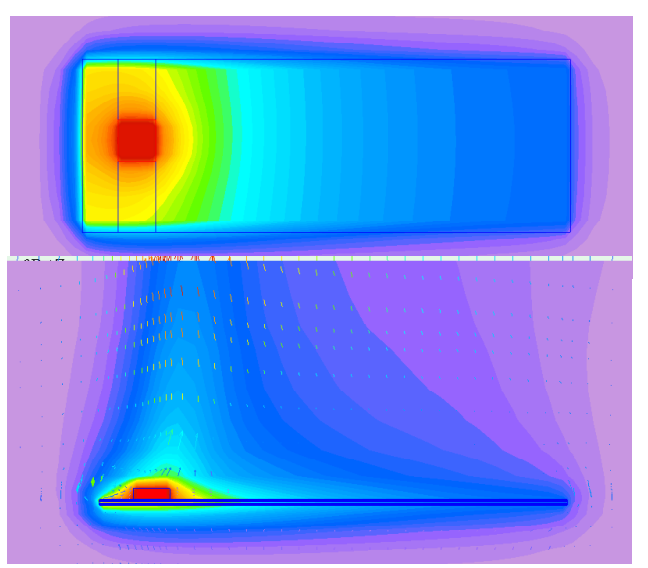

Fig. 4. Temperature distribution.

Table 2. Comparison between measured temperature and calculated temperature.

\begin{tabular}{lll}
\hline $\begin{array}{l}\text { Measurement } \\
\text { point }\end{array}$ & $\begin{array}{l}\text { Measured } \\
\text { temperature (c) }\end{array}$ & $\begin{array}{l}\text { Simulation } \\
\text { results (c) }\end{array}$ \\
\hline $\mathrm{A}$ & 44.3 & 43.4 \\
$\mathrm{~B}$ & 40.3 & 40.8 \\
$\mathrm{C}$ & 34.1 & 37.3 \\
$\mathrm{D}$ & 31.5 & 34.0 \\
$\mathrm{E}$ & 30.9 & 32.4 \\
$\mathrm{~F}$ & 28.7 & 30.2 \\
$\mathrm{G}$ & 27.3 & 28.3 \\
$T_{a m b}$ & 23.0 & 23.0 (given) \\
$T_{r e f}$ & 56.5 & 56.5 (given) \\
\hline
\end{tabular}

especially around the component. Comparing the two, it is observed that the numerical analysis result closely matches the measured data. On this result, this numerical analysis model has capability to reproduce the actual physical phenomenon.

\section{Parameter Study}

The parameters of the PCB structure that are thought to affect the thermal performance of the heat-dissipated component cooling are selected and given in Table 3. The outermost Copper layer (L1), the second Copper layer (P1) and the outermost insulation Solder Resist layer are focused because these close-to-the-air layers mainly affect the cooling. As the parameters, these layers' thickness, $\mathrm{Cu}$ remained ratio and board horizontal length are adopted. When one parameter is varied, the remaining parameters are fixed with the underlined value in Table 3.

4.1 Heat Dissipation The numerical simulation result of relationship between the heat-dissipation value and the reference 
Table 3. Parameters and their values.

\begin{tabular}{llllll}
\hline \hline $\begin{array}{l}\text { Heat dissipation (W) } \\
\text { Solder resist thickness }\end{array}$ & 0.5 & $\underline{\mathbf{1}}$ & 2 & 3 & \\
(um) & 20 & 30 & $\underline{\mathbf{4 0}}$ & 50 \\
$\begin{array}{l}\text { L1 Cu layer thickness } \\
\text { (um) }\end{array}$ & 10 & 20 & 30 & 40 & $\underline{\mathbf{4 8}}$ \\
$\begin{array}{l}\text { L1 Cu remained area } \\
\text { ratio }(\%)\end{array}$ & 10 & 20 & $\underline{\mathbf{3 0}}$ & 70 & 90 \\
$\begin{array}{l}\text { P Cu layer thickness } \\
\text { (um) }\end{array}$ & 10 & 20 & $\underline{\mathbf{3 5}}$ & 50 & 60 \\
$\begin{array}{l}\text { P1 Cu remained area } \\
\text { ratio }(\%)\end{array}$ & 10 & 30 & 50 & $\underline{\mathbf{8 0}}$ & 90 \\
Board length $(\mathrm{mm})$ & 40 & 60 & $\underline{\mathbf{8 4}}$ & 100 & \\
\hline
\end{tabular}

component temperature $T_{\text {ref }}$ is shown in Figure 5 .

This relation is described following linear expression, where $W$ is the heat-dissipation value of the component. $(0.5<\mathrm{W}<3.0)$

$$
T_{\text {ref }}=45.2 \times W+T_{a m b}
$$

The above equation tells that this system has 45.2 of Rjc (die-junction-to-case thermal resistance $(\mathrm{K} / \mathrm{W})$ between the component surface and the internal junction).

The specification of this component specifies that the junction temperature should be kept under 125 degree C. To make it possible, the temperature of the component surface has to be controlled lower than 80 degree $\mathrm{C}$ in this case.

The relationship as to the numerical analysis result between the distance from the center of the heat-dissipated component and the board surface temperature is shown in Figure 6.

For surface temperature distribution of each heat value, the temperature increases getting close to the component and it decreases apart from the component. At the point close to the component, surface temperature shows large gradient. When the heat value is increased, the surface temperature at each point is increased especially near the component.

Generally temperature gradient associates with thermal stress. The thermal stress at solder can be main cause of solder crack, then the graph also clarifies the importance of thermal management truly in case of high heat value.

Temperature distributions for each heat value cases are non-dimensionalized to find difference in its characteristic. This operation is to make surface temperature standardized from 0 to 1 that correspond to ambient temperature to $T_{\text {ref }}$. As a result, almost the same non-dimensionalized temperatures are obtained. In other words, any noticeable differences in surface temperature distribution were not found between them.

\subsection{Outermost Insulation Layer (Solder Resist)}

Thickness The relationship as to the numerical analysis result between Solder Resist thickness and $T_{\text {ref }}$ is shown in Figure 7.

The outermost solder resist thickness is varied from 10 to 50 $\mu \mathrm{m}$, but the components temperature $T_{\text {ref }}$ stays about 70 degree C. Each solder resist thickness case also shows similar PCB surface temperature distribution. The effect of cooling performance contributed by the outermost solder resist thickness is thought to be very little.

4.3 Outermost Copper Layer (L1) Thickness The relationship as to the numerical analysis result between outermost copper layer (L1) thickness and $T_{\text {ref }}$ is shown in Figure 8.

Component temperature $T_{\text {ref }}$ decreases if the L1 layer thickness decreased. $20 \mu \mathrm{m}$ and more is the thickness to keep $T_{r e f}$ lower than

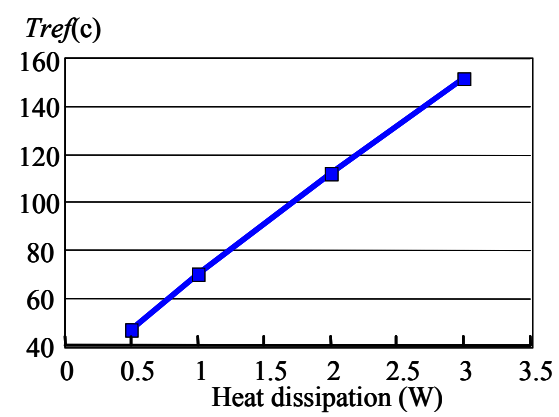

Fig. 5. Heat dissipation VS $T_{\text {ref. }}$

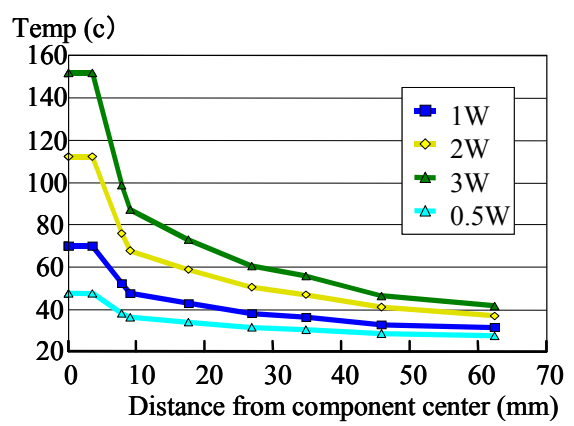

Fig. 6. Distance VS $T_{\text {ref }}$, depending on heat dissipation.

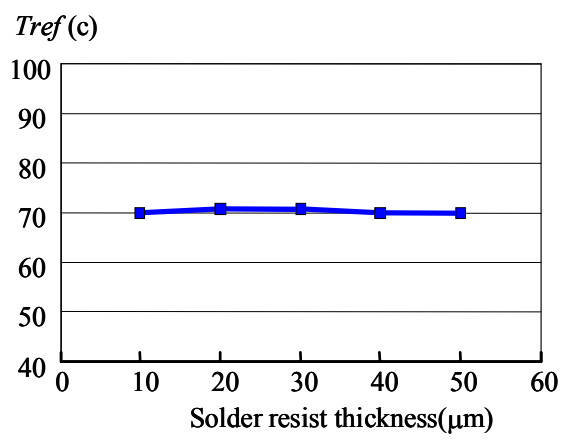

Fig. 7. Solder resist thickness VS $T_{\text {ref }}$.

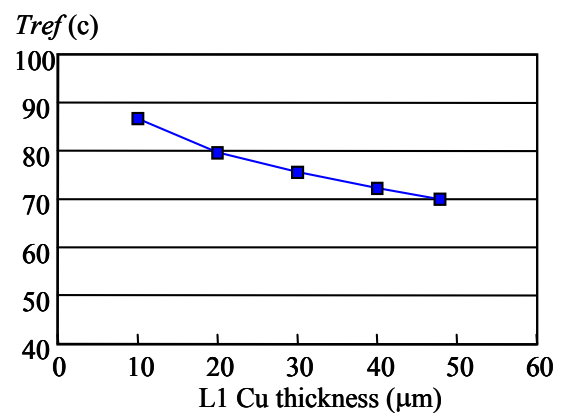

Fig. 8. L1 Cu thickness VS $T_{\text {ref }}$.

80 degree $\mathrm{C}$.

As the result, it is thought that the thickness of the outermost copper layer (L1) should be $20 \mu \mathrm{m}$ or more in this heat value $1(\mathrm{~W})$ case.

4.4 Copper Remained Ratio at Outermost Copper Layer (L1) The relationship as the numerical analysis result between the $\mathrm{Cu}$ remained ratio of the outermost copper layer (L1) and $T_{r e f}$ is shown in Figure 9. The analysis result shows that $T_{\text {ref }}$ decreases 
as $\mathrm{L} 1 \mathrm{Cu}$ remained ratio increases. $T_{\text {ref }}$ crosses 80 degree $\mathrm{C}$ when the $\mathrm{Cu}$ remained ratio is $10 \%$, therefore $10 \%$ is the minimum preferable $\mathrm{Cu}$ remained ratio to keep $T_{\text {ref }}$ below 80 degree $\mathrm{C}$ in this $1(\mathrm{~W})$ component case. Another noticeable point is that the $T_{\text {ref }}$ shows remarkable decrease if the $\mathrm{Cu}$ remained ratio falls at the range of 10 to $30 \%$.

The relationship of the numerical analysis result between the distance from the center of the heat-dissipated component and the board surface temperature is shown in Figure 10. The figure shows that surface temperature decrease near the component, and increase at the end of the card in accordance with increasing of $\mathrm{Cu}$ remained ratio. The difference in surface temperature, depends on $\mathrm{Cu}$ remained ratio, is remarkable close to the component. In other words, the higher $\mathrm{Cu}$ remained ratio card plays important role to transfer the heat to wide area of the PCB.

4.5 Second Copper Layer (P1) Thickness The relationship as to the numerical analysis result between second copper layer (P1) thickness and $T_{\text {ref }}$ is shown in Figure 11. The same trend as L1 layer is observed.

4.6 Copper Remained Ratio at Second Copper Layer (P1) The relationship as to the numerical analysis result between the $\mathrm{Cu}$ remained ratio of the second copper layer (P1) and $T_{\text {ref }}$ is shown in Figure 12. The relationship as to the numerical analysis result between the distance from the center of heat-dissipated component and the board surface temperature is shown in Figure 13. These $T_{\text {ref }}$ and surface temperature distribution of each $\mathrm{P} 1 \mathrm{Cu}$ remained area cases show the same trend as L1 outermost copper layer. However they have less dependency on $\mathrm{Cu}$ remained ratio than those of $\mathrm{L} 1 \mathrm{Cu}$ remained ratio cases.

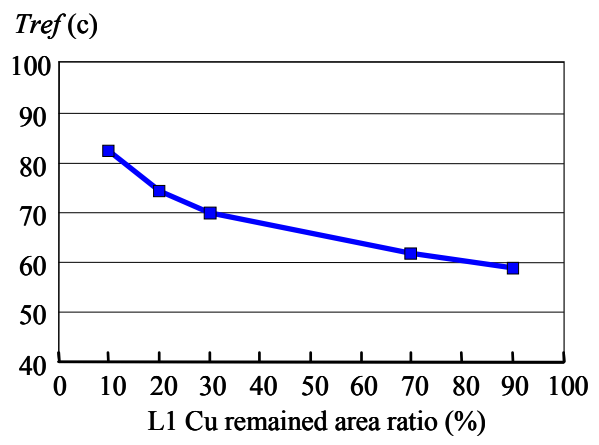

Fig. 9. L1 Cu remained area ratio VS $T_{\text {ref. }}$.

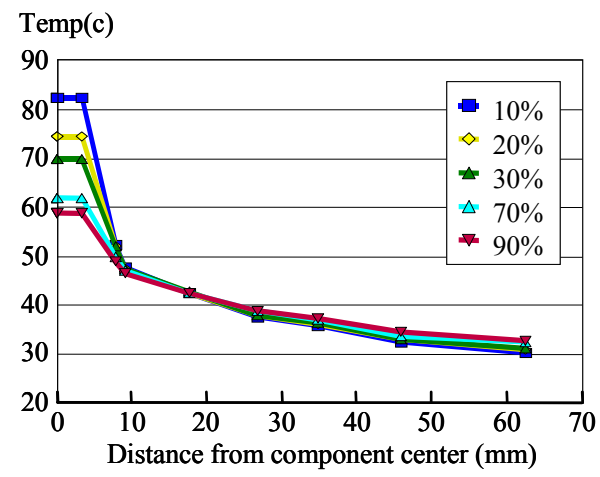

Fig. 10. Distance VS surface temperature, depending on $\mathrm{L} 1 \mathrm{Cu}$ remained area ratio.
4.7 Board Horizontal Length The relationship of the numerical analysis result between the $T_{\text {ref }}$ and board horizontal length is shown in Figure 14. The $T_{r e f}$ decreases when board length

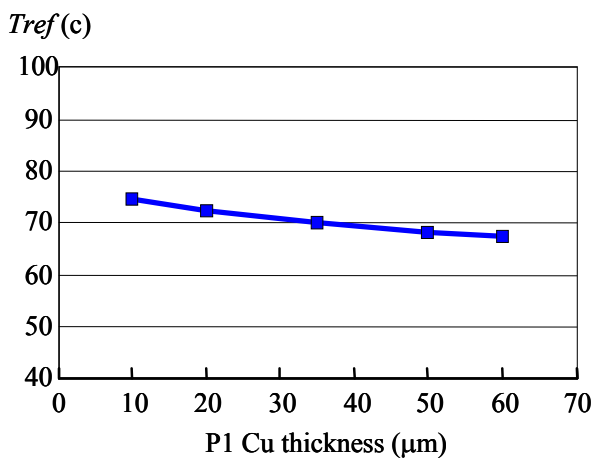

Fig. 11. P1 Cu thickness VS $T_{\text {ref. }}$.

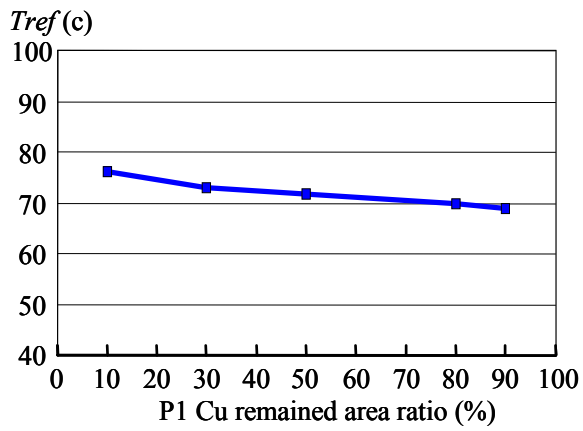

Fig. 12. P1 Cu remained area ratio VS $T_{\text {ref. }}$.

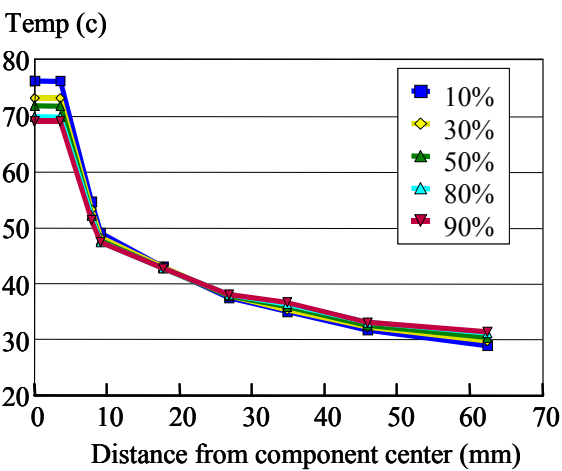

Fig. 13. Distance VS surface temperature, depending on $\mathrm{P} 1 \mathrm{Cu}$ remained area ratio.

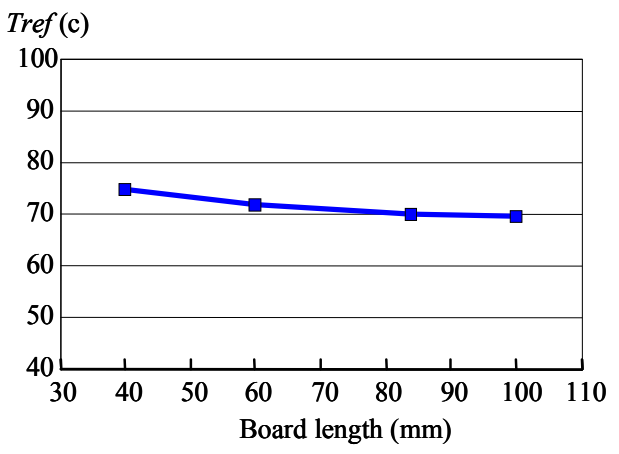

Fig. 14. Board horizontal length VS $T_{\text {ref. }}$ 
is increased to about $70 \mathrm{~mm}$ but it stays when the board length exceeds $70 \mathrm{~mm}$ in this case. The idea enlarging the PCB seems very simple and useful so as to decrease the component temperature in one sense, however the idea only works within a certain board length. In other words, the above implies importance of the temperature difference distribution between the fluid and heat dissipated component. This parameter study in section 4 unveiled the important fact that optimizing the $\mathrm{Cu}$ layer thickness and $\mathrm{Cu}$ remained ratio lead to high performance thermal management.

It is very hard to tune these many factors simultaneously to capture the best condition. Dimensional analysis and non-dimensional numbers that represent those factors are introduced in section 5 .

\section{Non-Dimensional Numbers Correlation}

5.1 Dimension Analysis Dimensional analysis is widely used to evaluate convection of solid and fluid system, because heat flux through the convection interface is affected by many factors such as physical properties of the fluid, fluid status and solid shape.

Generally, natural air convection heat transfer is described with dimensionless number below ${ }^{(3)(4)}$.

$$
N u=f(P r, G r)
$$

$N u$ : Nusselt number, Pr: Prandtl number, Gr: Grasshoff number $\mathrm{Nu}, \mathrm{Pr}, \mathrm{Gr}$ are described below equations.

$$
\begin{aligned}
& N u=\frac{h l}{k} \\
& \operatorname{Pr}=\frac{v}{\alpha} \\
& G r=\frac{\beta g \theta l^{3} \rho^{2}}{\mu^{2}}
\end{aligned}
$$

$h$ : heat convection coefficient $\left(\mathrm{W} / \mathrm{m}^{2} \mathrm{~K}\right)$

$l$ : reference length $(\mathrm{m})$

$k$ : thermal conductivity $(\mathrm{W} / \mathrm{mK})$

$v:$ kinetic viscosity $\left(\mathrm{m}^{2} / \mathrm{s}\right)$

$\alpha:$ thermal diffusivity $\left(\mathrm{m}^{2} / \mathrm{s}\right)$

$\beta$ : buoyancy effect $(1 / \mathrm{K})$

$g:$ gravitational acceleration $\left(\mathrm{m} / \mathrm{s}^{2}\right)$

$\theta:$ temperature difference $(\mathrm{K})$

$\rho:$ density $\left(\mathrm{kg} / \mathrm{m}^{3}\right)$

$\mu$ : viscosity $(\mathrm{kg} / \mathrm{ms})$

5.2 Derivation of Non-Dimensional Number in PCB

Cooling To apply dimensional analysis to PCB cooling system, some unique ideas are required to derivate non-dimensional numbers. The following four ideas enable to derive dimensionless numbers that represent the PCB properties and cooling system.

1) The temperature difference in $\mathrm{Gr}$ is the difference of ambient temperature and component temperature.

2) The reference length $l$ in $\mathrm{Nu}$ and $\mathrm{Gr}$ is a radius length of the circle area where the non-dimensionalized temperature is above 0.5 . The definition of non-dimensional temperature $T^{*}$

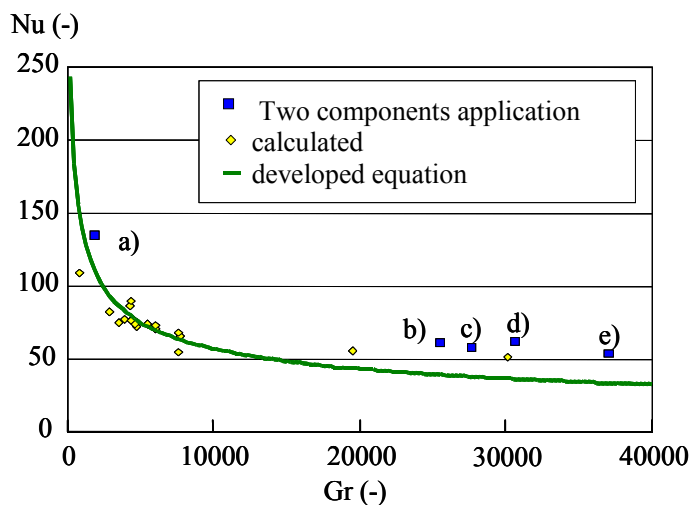

Fig. 15. Comparison of calculated $\mathrm{Nu}-\mathrm{Gr}$ and developed equation.

is derived following.

$T^{*}=\frac{T-T_{a m b}}{T_{r e f}-T_{a m b}}$

3) Specific heat, kinetic viscosity, density, thermal conductivity, in $\mathrm{Pr}$ are to be obtained at the temperature of arithmetic mean of component and ambient.

4) $\mathrm{Nu}$ is derived from the following equation.

$$
N u=\frac{W}{k L\left(T_{\text {ref }}-T_{\text {amb }}\right)}
$$

The equation above is derived following way. It is well known that transferred heat value $Q$ between fluid and particle is described below as the Newton's law.

$$
\begin{aligned}
& Q=A h\left(\theta_{p}-\theta_{f}\right) \\
& A: \operatorname{area}\left(\mathrm{m}^{2}\right)
\end{aligned}
$$

In steady stats, the heat dissipating from PCB to air equals to heating value $W$ of the component. The effective area $A_{e}$ is introduced that is a area considered to work for heat transfer, then the previous equation is described below.

$$
Q=A_{e} h\left(\theta_{p}-\theta_{f}\right)=W
$$

$A_{e}$ : effective area

$A_{e}$ is considered as a circle area which radius is the reference length defined above.

5.3 Non-dimensional Numbers Correlation Figure 15 shows obtained sets of $\mathrm{Nu}$ and $\mathrm{Gr}$. These sets of non-dimensional numbers are the result of parameter study in section 4 . The Solid line in the figure represents the estimated correlation of $\mathrm{Nu}$ and $\mathrm{Gr}$. The power coefficient in equation of the correlation equation is calculated to fit the parameter study. The estimated non-dimensional correlation is given below.

$$
N u=80000(G r \cdot P r)^{-\frac{2}{5}}
$$

\section{Discussion}

The parameter study in section 4 leads to a guideline to get better cooling for the specific PCB. That is to make the outer layers thickness and $\mathrm{Cu}$ remained ratio increased.

However, the heat convection in PCB cooling is not simple and many factors are to be taken into consideration, so it is hard to generalize it. 


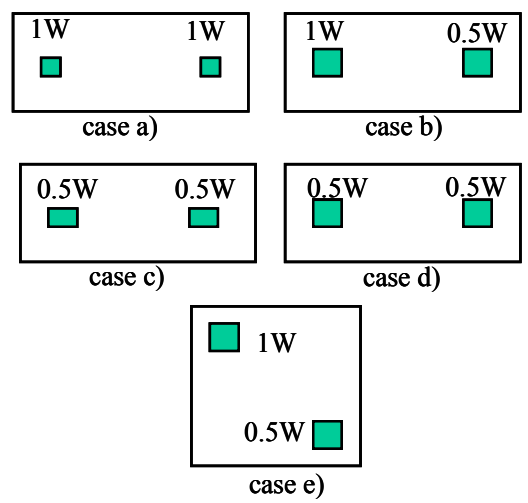

Fig. 16. Two components application study.

If the heat value of the component is too large, it would be no use to thicken the $\mathrm{Cu}$ layer thickness to get better cooling. In such condition, temperature distribution should be checked first and study the how large area contributes to the cooling. So, it is highly recommended to introduce non-dimensional Numbers that can represent various physical properties.

The below two ideas performed in estimating dimensionless numbers enables to express the heat convection in cooling PCB.

1) Reference length represents the temperature distribution.

2) Effective area represents the effective area where the heat transfer is performed.

To discuss the application of the derived correlation, numerical analysis is performed to reproduce five PCB cooling system that has two components. Figure 16 shows the geometry of the components on each PCB. Case a) to d) are the same PCB size as that in section 3 and case e) is a square of $100 \times 100(\mathrm{~mm})$. At the same time the $A_{e}$ and $l$ are re-defined below to cover multi heat dissipation components cooling system. $A_{e}$ is the total area where the $T^{*}$ is above 0.5 . And $l$ is the radius of the circle which area is $A_{e}$. The results of the numerical analysis are processed to calculate $\mathrm{Nu}$ and $\mathrm{Gr}$ as explained in section 5. The calculated sets of dimensionless numbers are plotted as square mark in figure 15.

The result shows that $\mathrm{Nu}$ and $\mathrm{Gr}$ of the two components system are appeared on the same curve of those of one component system. So, it can be said this application and ideas to derive $\mathrm{Nu}$ and $\mathrm{Gr}$ are useful and justified.

\section{Conclusion}

In this paper, heat convection from component on $\mathrm{PCB}$ to the air is evaluated to have effective cooling.

Increasing the thickness and $\mathrm{Cu}$ remaining ratio of the outside $\mathrm{Cu}$ layer can help cooling the PCB.

Temperature distribution of the $\mathrm{PCB}$ and effective area are to be take into consideration to evaluate PCB cooling.

To estimate non-dimensional numbers based on the previous ideas, reference length can be obtained with no-dimensionalized temperature distribution and effective area can be with square of the reference length.

These ideas bring up a dimensionless numbers correlation below that reproduces the heat transfer.

(Manuscript received Dec, 24, 2002)

\section{References}

(1) T.Nakanishi and T.Nishio : "Optimal Thermo-Structural Analysis for CSP/FCA/MCM Mounting on Build-up Board", Proceedings of the $3^{\text {rd }}$ International Conference on Benefiting from Thermal and Mechanical Simulation in (Micro)-Electronics EuroSIME 2002, Paris, France, April 15-17, pp.369-376 (2002)

(2) K.Shimohashi and T.Nakanishi : "Development of Dimensionless Correlation for Natural Convection Cooling Board", Proceedings of the $3^{\text {rd }}$ International Conference on Benefiting from Thermal and Mechanical Simulation in (Micro)-Electronics EuroSIME 2002, Paris, France, April 15-17, pp.176-181 (2002)

(3) K.Nishikawa and N. Kitayama : "Thermal Engineering", Ohm publishing (1982)

(4) T. Kunii et. al. : Transport phenomena, Baifukan publishing (1992)

Tohru Nakanishi (Non-member) joined IBM in 1987, starting

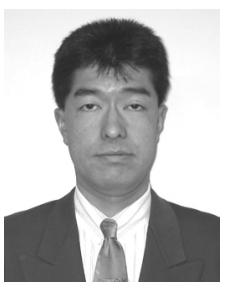

Government with the design and evaluation of the components to be used for the large computer system. At present, he is providing the best solution for the Structural and Thermal problem with the numerical analysis. He is a Professional Engineer for Applied Physical Science, authorized by the Japanese

Koki Shimohashi (Non-member) received Master's degree of

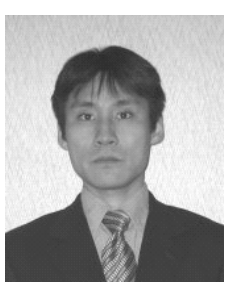
Materials Science and Engineering from Kyushu University in 1998. After graduation he works for IBM as an Engineer. In IBM, He is focusing on new PCB design evaluation in the standpoints of thermal stress and electronic signal quality.

Tomohiro Hase

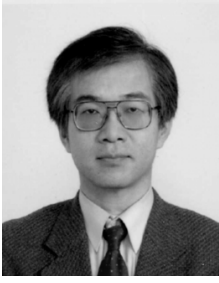

(Member) was born in 1953 in Gifu, Japan. Received a B.S.degree from Fukui University, and an M.S.degree and Ph.D.degree from Shizuoka University, Japan, all degrees were in electronics engineering, in 1977, 1979 and 1995. In 1979, he joined the Mitsubishi Electric Corporation, and engaged in research into signal processing for audio visual equipments and microprocessor application. In 2002, he is a Professor of the Faculty of Science and Technology, Ryukoku University. 